CORRECTION OF THE TRAJECTORIES IN THE BOOSTER

$$
\text { PROTON INJECTION LINE: a MODEL-BASED STUDY }
$$

\author{
AD \\ BOOSTER TECHNICAL NOTE \\ NO. 139
}

F. Z. KHIARI and A. U. LUCCIO

MAY 19, 1989

ACCELERATOR DEVELOPMENT DEPARTMENT

BROOKHAVEN NATIONAL LABORATORY

UPTON, NEW YORK 11973 


\title{
Correction of the trajectories in the Booster Proton Injection Line: a Model-Based Study
}

\author{
F. Z. Khiari, A. U. Luccio \\ Brookhaven National Laboratory \\ Upton, $N Y 11973$
}

\section{INTRODUCTION}

In order to ease and speed up the commissioning period of the AGS Booster, it is necessary to identify potential problems well ahead of time and to find solutions. One section of the accelerator complex whose priority is high on the agenda is the new proton injection line from the Linac into the Booster. In this note, we present a computer-model $\left(\mathrm{MAD}^{[1]}\right)$ study of the line, evaluate the effects of systematic and random errors in the magnets on the beam trajectory and develop algorithms to correct the distorted orbit. These algorithms are successfully tested by the model.

\section{PROTON INJECTION LINE}

The Booster proton injection line is designed ${ }^{[2]}$ to transport $H^{-}$ion beams from tank 9 of the $200 \mathrm{MeV}$ Linac and inject them into the Booster ring. A layout of the line is shown in Fig. 1. A fast pulsed kicker magnet deflects the $H^{-}$beam by $7.5^{\circ}$ from the previous HEBT line into the new proton injection line. A series of 4 identical bending magnets, powered from a single power supply, takes the beam through a $126^{\circ}$ bend into the last section of the line before injection into the Booster ring. The focussing of the beam in the transverse plane is achieved by 13 quadrupoles. The beam position along the line are read from 7 beam position monitors ${ }^{[3]}$. Each monitor gives both the horizontal and the vertical position of the beam. A set of two beam profile monitors ${ }^{[3]}$ are used to determine the beam widths. Three d.c. horizontal kicker magnets and four d.c. vertical kicker magnets are used to correct the distorted beam trajectory. A fast pulsed vertical steering 
magnet is used for transverse (vertical) painting. The optics of the line is determined by matching ${ }^{[2]}$ the (input) horizontal beam phase-ellipse form tank 9 of the Linac to the horizontal acceptance of the Booster ring at the injection point. This matching point is situated in the middle of the first (horizontal) quadrupole after the main ring dipole injection magnet. The matching is done for both the high intensity (unpolarized) and low intensity (polarized) modes of operation.

\section{SYSTEMATIC ERRORS IN THE PROTON INJECTION LINE}

\section{Systematic Field Errors and Proton Energy Spread}

The 4 bending dipoles are powered from a single power supply. An error in the set point of this power supply will produce a systematic field error in the bending magnets. The $200 \mathrm{MeV}$ beam delivered by the Linac has an energy spread of $\pm 0.2 \%{ }^{[4]}$, i.e. $\Delta T=$ $\pm 0.4 \mathrm{MeV}$, where $T$ is the kinetic energy of the beam. To find the beam trajectories for the extreme energies, $T \pm \Delta T$, we simulate the energy difference $\Delta T$ by an additional dipole field error $\Delta B$ given by

$$
\Delta B=\beta^{2} B \times \frac{\Delta T}{T}
$$

where $B$ is the dipole field corresponding to the particles whose kinetic energy is $T$ and whose speed (in units of the speed of light) is $\beta$. As an illustration, we took $\Delta B / B=-0.1 \%$ and studied the combined effect of the energy spread and the systematic field error in the bending dipole magnets. Fig. 2 shows:

(a) a linear plot of the line elements along with a plot of $\beta_{x}, \beta_{y}$ (dashed line) and the horizontal dispersion, $X_{p}$,

(b) the (horizontal) beam envelope along the line illustrating the effect of the energy spread in the line and

(c) the combined effect of the energy spread and systematic field errors in the dipoles. 


\section{RANDOM ERRORS IN THE PROTON INJECTION LINE \\ $\underline{\text { Random Position, Orientation and Field Errors }}$}

We studied the effect of random errors on the beam trajectory in the proton injection line. We assumed that the random errors on the transverse $(x, y)$ and longitudinal (s) positions of the magnets, dipoles and quadrupoles, are uniformly distributed and fall within the range $\pm 0.2 \mathrm{~mm}$. The errors on the three orientations of the magnets were also assumed uniformly random and within the interval $\pm 0.5 \mathrm{mrad}$. The random relative field errors in the dipoles were also uniformly random in the range $\pm 0.1 \%$ and those in the quadrupoles in the interval $\pm 0.5 \%$. Several cases were studied, each one corresponding to a different seed for the extraction of random errors.

\section{CORRECTIONS OF THE DISTORTED ORBIT IN THE PROTON INJECTION LINE $\underline{\text { RMS Correction }}$}

The principle of the rms correction is to minimize the rms deviation of the distorted orbit from the design trajectory. The input to the correction algorithm consists of the model-simulated values of

i) the beam positions at the beam position monitors,

ii) the beta functions and phase advances at the locations of the beam position monitors and the kickers.

The algorithm used to calculate the kicks that minimize the distortion of the orbit was based on the Householder transformation ${ }^{[5]}$. This transformation, needed because the number of beam position monitors is greater than the number of kickers, allows us to optimize in a least square sense the centering of the orbit in the beam position monitors.

Let $X_{i}(\mathrm{x}$ or $\mathrm{y}), i=1, \ldots, m$, be the displacement of the orbit at BPM \# $i$ before correction and $T_{i j} \theta_{j}$ the displacement of the orbit at BPM \# $i$ due to a kick $\theta_{j}$ in kicker $\# j, j=1, \ldots, n$. We define the norm 


$$
S \equiv \sum_{i=1}^{m} \sum_{j=1}^{n}\left(X_{i}+T_{i j} \theta_{j}\right)^{2}
$$

where

$$
\begin{gathered}
T_{i j}=\sqrt{\beta_{i} \beta_{j}} \sin \left(\phi_{i}-\phi_{j}\right), \quad s_{i} \geq s_{j} \\
T_{i j}=0, \quad s_{i} \leq s_{j}
\end{gathered}
$$

where $s$ is the distance along the line. The kicks $\theta_{j}$ that minimize the orbit distortion are found by solving the system of equations

$$
\frac{\delta S}{\delta \theta_{j}}=0
$$

The vector kick $\vec{\theta}$ is given in terms of the vector displacement $\vec{X}$ and the response matrix $T$ by

$$
\vec{\theta}=-\left(T^{R} T\right)^{-1} \times T^{R} \vec{X}
$$

where $T^{R}$ is the transpose of $T$. The matrix $T$ is not square and the matrix $T^{R} T$ is highly singular and generally is not invertible. Thus, instead of using Eq. (5), the system of equations (4) is solved with the help of the Householder transformation.

Notice that, even though this method reduces the rms distortion of the orbit, sometimes the corrected orbit is left with a sizable displacement at the matching point. A way to deal with this problem is presented in the following section.

\section{Centering the Orbit at the Last Two BPM's}

As was seen in the previous section, the displacement and angle of the corrected orbit at the matching point can be large. One way to reduce them is to add extra kicks at the last two kickers to center the orbit at the last two beam position monitors. We should 
point out that this method of correction can be used by itself or in conjunction with the previous one. In the latter case, the centering of the orbit has to be done after the rms correction.

Let $X_{m-1}$ and $X_{m}$ be the displacements of the orbit at the last two BPM's after the rms correction. The kicks $\theta_{n-1}$ and $\theta_{n}$ at the last two kicker magnets that center the orbit are given in matrix form by

$$
\vec{\theta}=-T^{-1} \vec{X}
$$

where $T$ is the response matrix referred to earlier. Fig. 3 shows:

(a) a distorted orbit due to random position, orientation and field errors,

(b) the previous distorted orbit with the rms correction, and

(c) the distorted orbit with the rms correction and the centering at the last two beam position monitors.

Notice that both the displacement and angle of the distorted orbit at the matching point were reduced in case (c) from their values in case (b).

Figs. 4 and 5 illustrate the cases of Figs. 2 and 3 for the polarized proton beam.

\section{CONCLUSION}

We successfully tested two orbit correction schemes for the model-based Booster proton injection line. The algorithms are automated and can be used in conjunction with manual steering during the commissioning period of the real injection line.

\section{ACKOWLEDGEMENTS}

We have benefitted from discussions with a number of people. We would like to thank C. Gardner, R. Gupta, Y.Y. Lee, K. Reece, A. Soukas and R. Witkover for useful discussions. 


\section{REFERENCES}

[1] F. C. Iselin \& J. Niederer, The MAD Program, Version 6, CERN/LEP-TH/87-33, the data were supplied by R. Gupta.

[2] Booster Design Manual, pp. 4.1-4.10

[3] Booster Design Manual, pp. 8.1-8.7

[4] J. Alessi \& M. Brennan, Private Communication

[5] The Householder Transformation is a SLATEC Routine, See also W. H. Press et al, Numerical Recipes, pp. 59 


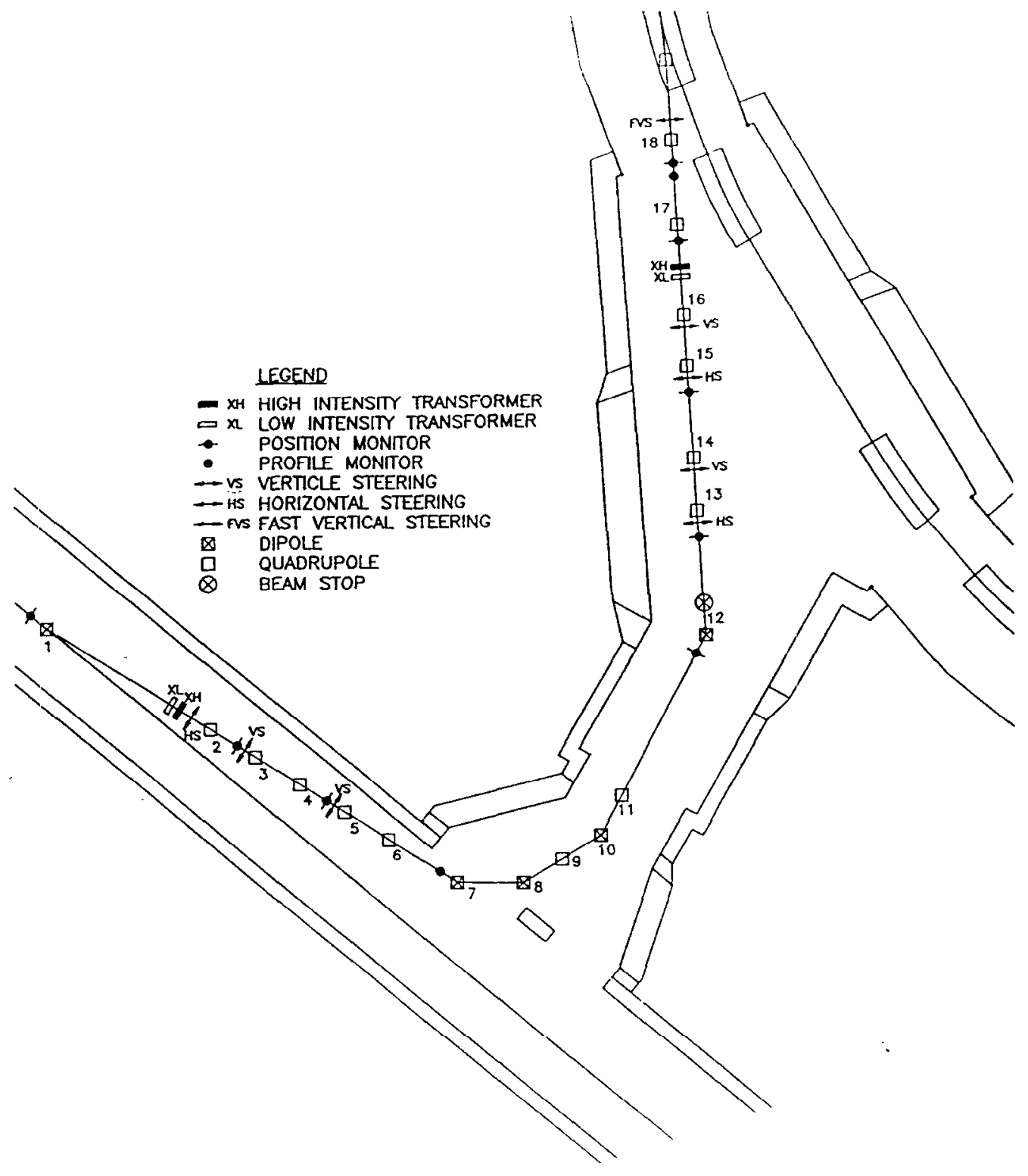

Fig. 1 


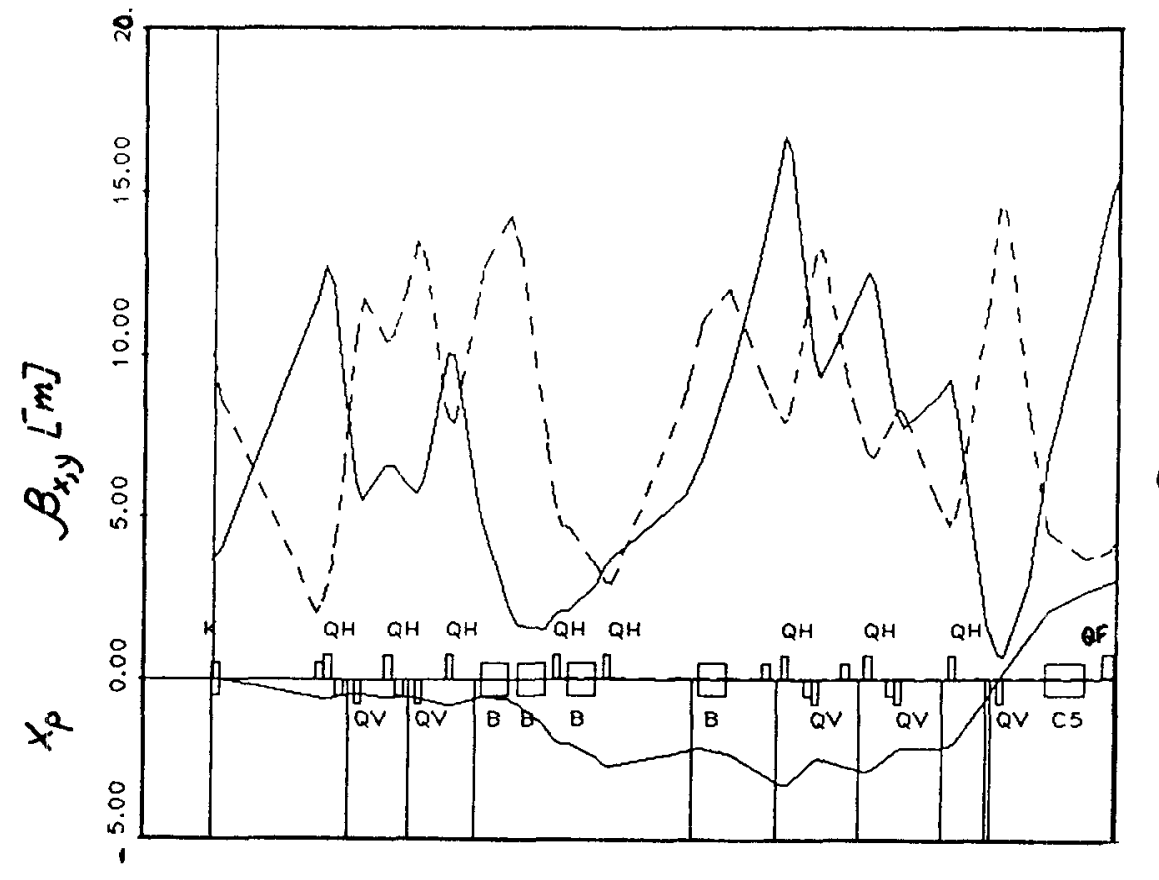

(a)
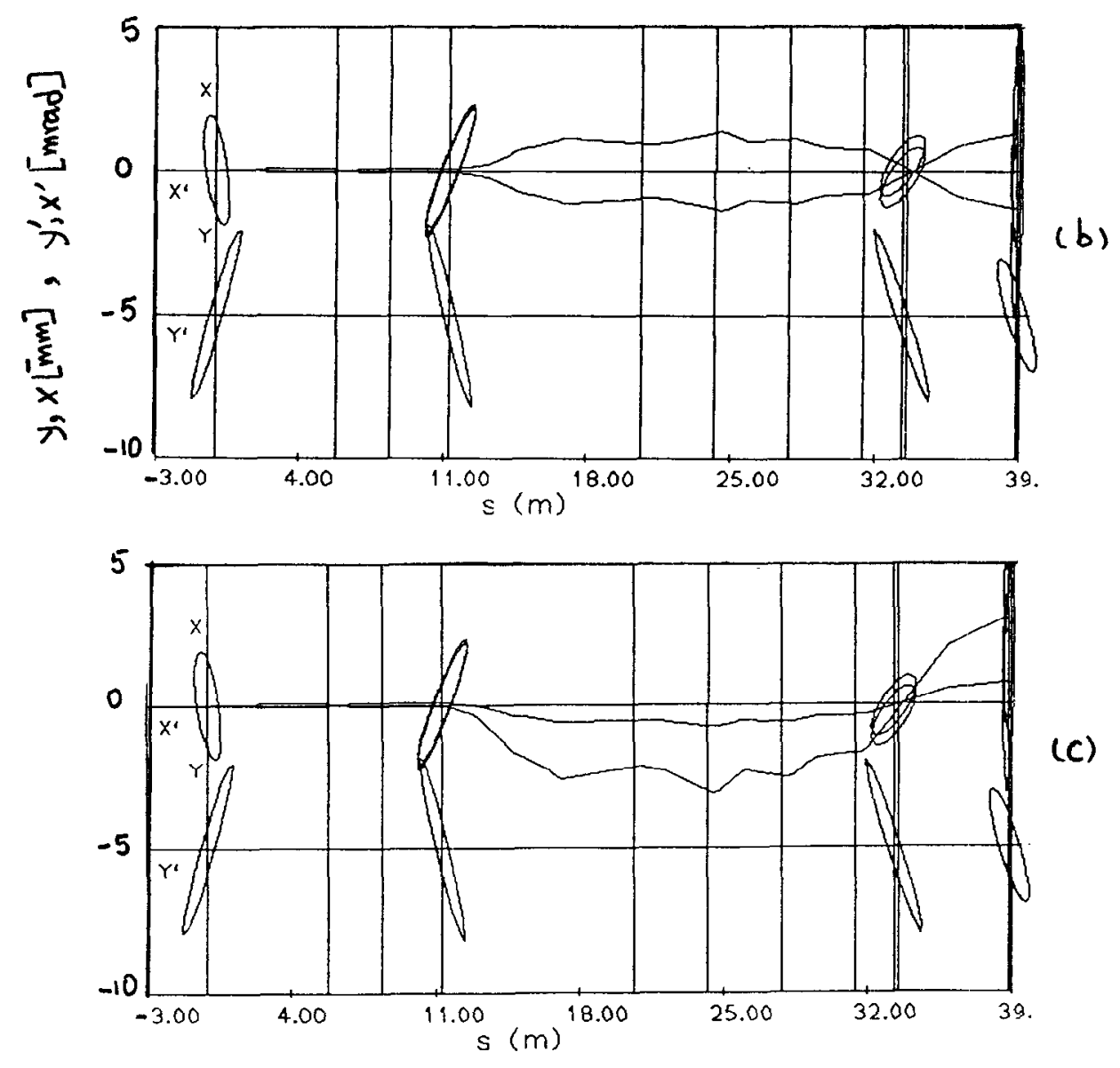

Fig. 2 


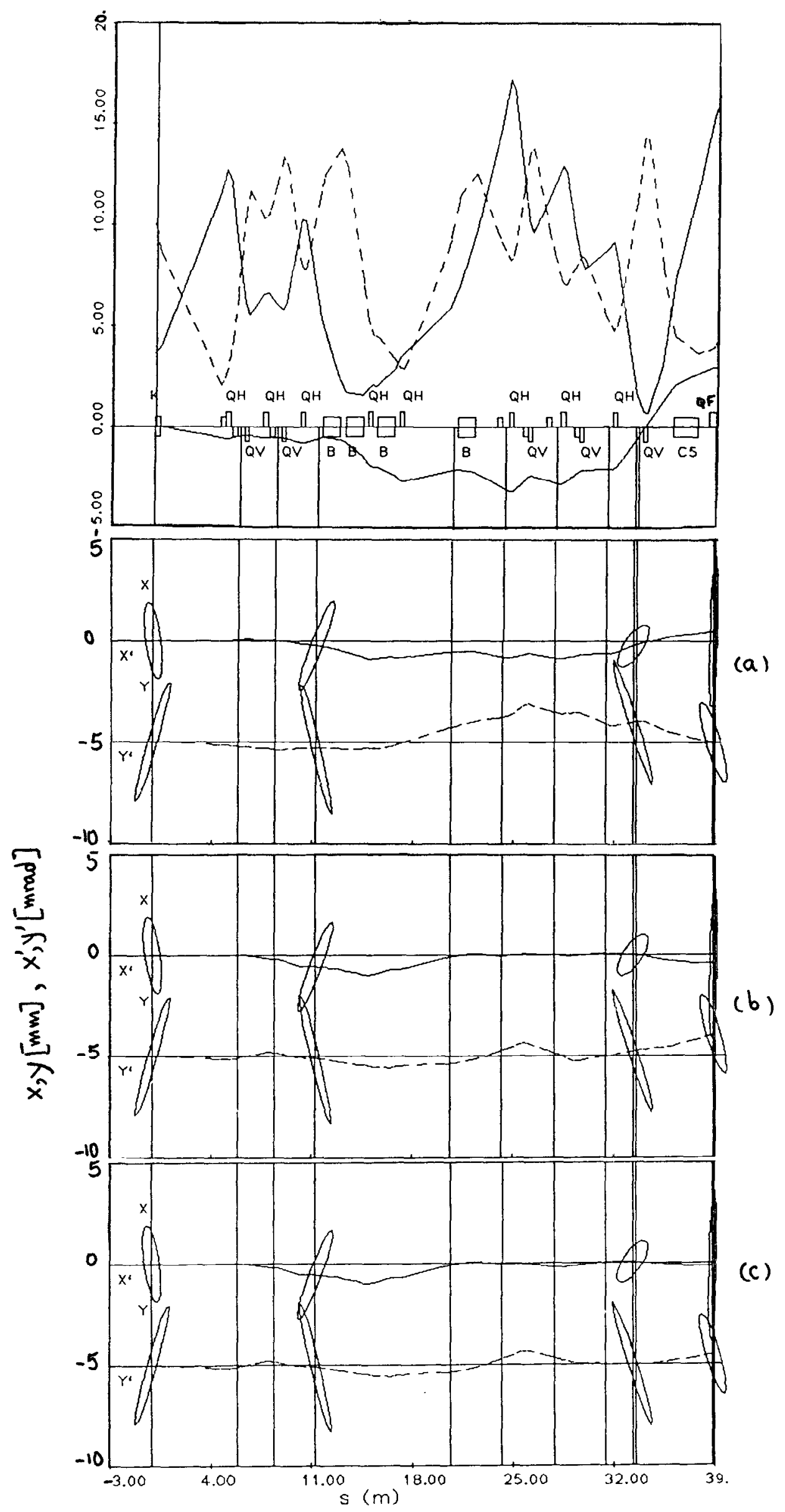

Fig. 3 


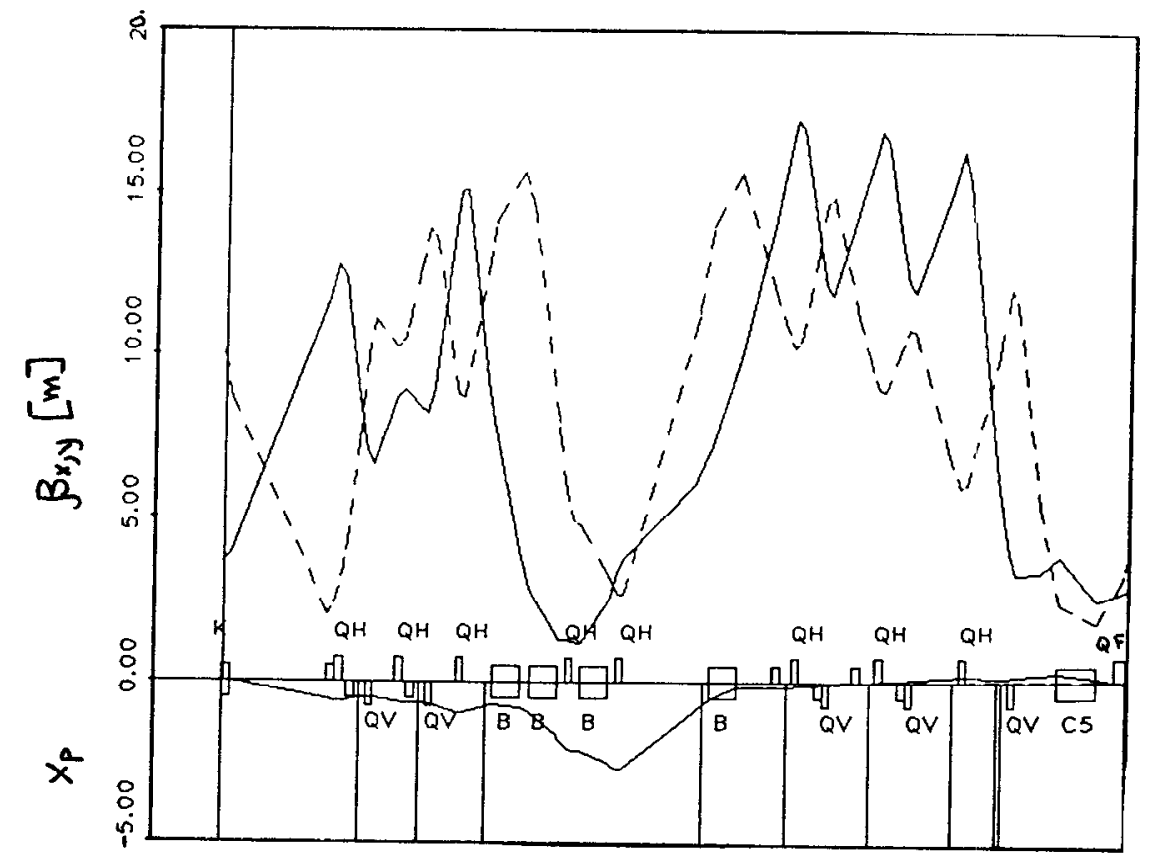

(a)

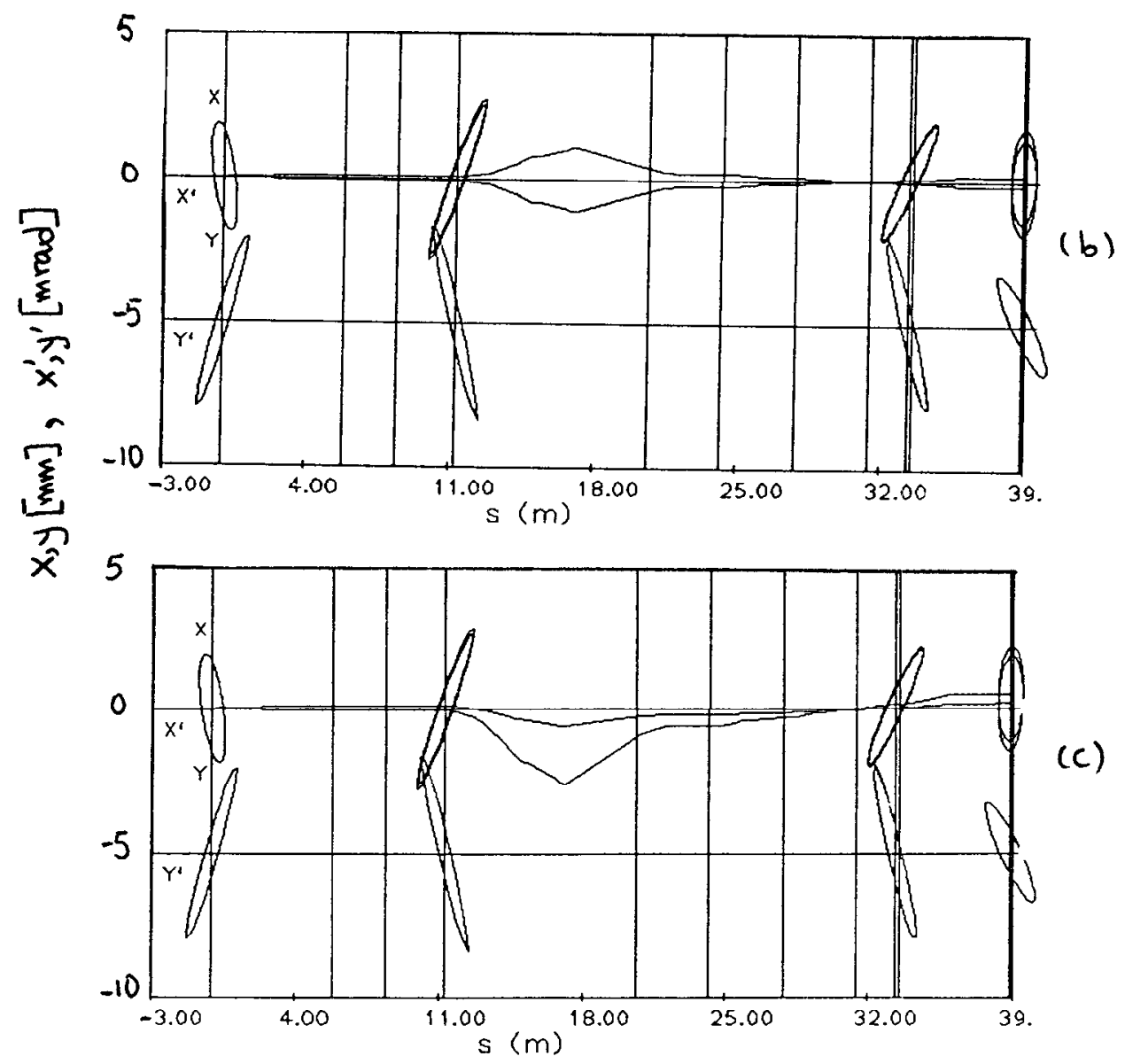

Fig. 4 


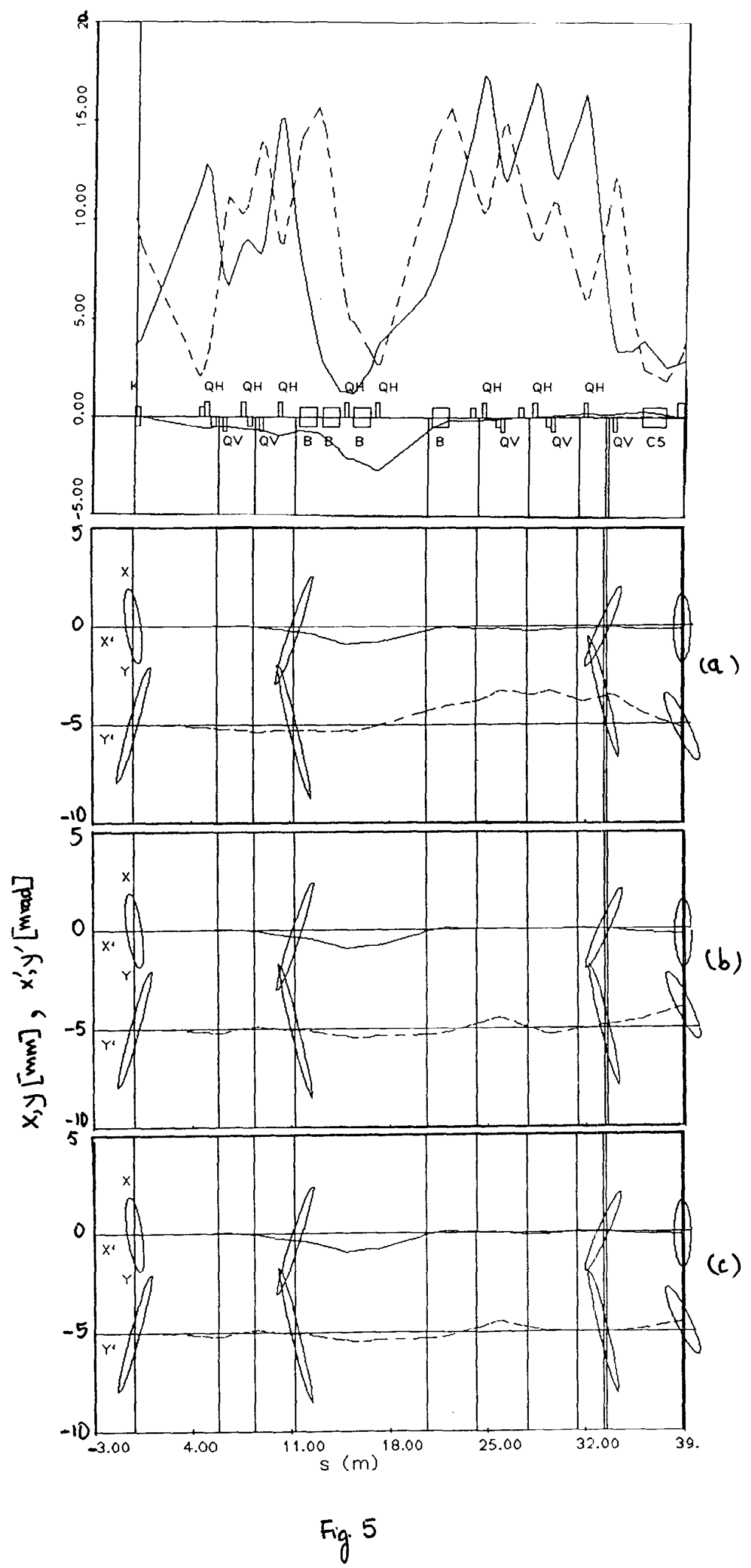

